\title{
Percutaneous Vertebroplasty: Preliminary Experiences with Rotational Acquisitions and 3D Reconstructions for Therapy Control
}

\author{
Roman Hodek-Wuerz, ${ }^{1}$ Jean-Baptiste Martin, ${ }^{2}$ Kai Wilhelm, ${ }^{2, *}$ Karl O. Lovblad, ${ }^{2}$ \\ Drazenko Babic, ${ }^{3}$ Daniel A. Rufenacht, ${ }^{2}$ Stefan G. Wetzel ${ }^{1}$ \\ ${ }^{1}$ Department of Radiology, Section of Neuroradiology, University Hospital Basel, Basel, Switzerland \\ ${ }^{2}$ Department of Radiology, Section of Neuroradiology, University Hospital Geneve, Geneva, Switzerland \\ ${ }^{3}$ Philips Medical System, Best, The Netherlands
}

\section{Abstract}

Percutaneous vertebroplasty (PVP) is carried out under fluoroscopic control in most centers. The exclusion of implant leakage and the assessment of implant distribution might be difficult to assess based on two-dimensional radiographic projection images only. We evaluated the feasibility of performing a follow-up examination after PVP with rotational acquisitions and volumetric reconstructions in the angio suite. Twenty consecutive patients underwent standard PVP procedures under fluoroscopic control. Immediate postprocedure evaluation of the implant distribution in the angio suite (BV 3000; Philips, The Netherlands) was performed using rotational acquisitions (typical parameters for the image acquisition included a $17-\mathrm{cm}$ fieldof-view, 200 acquired images for a total angular range of $180^{\circ}$ ). Postprocessing of acquired volumetric datasets included multiplanar reconstruction (MPR), maximum intensity projection (MIP), and volume rendering technique (VRT) images that were displayed as two-dimensional slabs or as entire three-dimensional volumes. Image evaluation included lesion and implant assessment with special attention given to implant leakage. Findings from rotational acquisitions were compared to findings from postinterventional CT. The time to perform and to postprocess the rotational acquisitions was in all cases less then $10 \mathrm{~min}$. Assessment of implant distribution after PVP using rotational image acquisition methods and volumetric reconstructions was possible in all patients. Cement distribution and potential leakage sites were visualized best on MIP images presented as slabs. From a total of 33 detected

*Present address: Department of Radiology, University Hospital of Bonn, Bonn, Germany.

Correspondence to: R. Hodek-Wuerz; email: hodekr@uhbs.ch leakages with CT, 30 could be correctly detected by rotational image acquisition. Rotational image acquisitions and volumetric reconstruction methods provided a fast method to control radiographically the result of PVP in our cases.

Key words: Spine-Complication-Interventional radiology_-Vertebroplasty_-3D rotational imaging

Percutaneous vertebroplasty (PVP) is a procedure that entails percutaneous injection of polymethyl methacrylate (PMMA), or cement, into a diseased vertebra. First described in 1987 for the treatment of aggressive vertebral hemangioma [1], the main indications have been extended to the treatment of painful osteoporotic compression fractures, vertebral myeloma, and metastases [2-4].

In most centers, all phases in the procedure of PVP are usually performed under fluoroscopic digital subtraction angiogography (DSA) control [5]. As, in principle, needle insertion can be performed under computed tomography (CT) guidance [6], the fluoroscopically controlled accessin most, transpedicular-is reasonable, as it is fast to accomplish and safe in experienced hands. For the second step, the cement injection, the fluoroscopic control is mandatory in order to avoid cement extravasation into the paravertebral spaces or veins. Whereas for the first two steps fluoroscopic images are the method of choice, for the third step - the final assessment of implant distribution-CT can be considered the method of choice. CT allows the acquisition of volumetric datasets and, thus, an exact assessment of implant distribution. This might be of interest especially in the treatment of metastasis and-more important-to search for cement extravasation into critical structures, such as veins at the vertebral foramen, if findings from twodimensional (2D) fluoroscopic images are equivocal. 
With the introduction of rotational image acquisition, it became possible to acquire high-resolution angiographic three-dimensional (3D) image [7, 8]. However, it is possible to employ this technique to other than vascular areas. We sought to evaluate the feasibility to assess implant distribution and potential leakage sites after standard PVP procedures with 3D rotational acquisitions.

\section{Materials and Methods}

\section{Patients and PVP Procedure}

Twenty consecutive patients ( 8 female, 12 male; mean age: 67 years; age range: 49-85 years) who underwent standard PVP procedures under fluoroscopic control with rotational acquisition for immediate postprocedural control and who had additionally undergone a clinically indicated CT examination in the postintervention period (within 1 week) were evaluated. The main indications for PVP were metastasis $(n=12)$, osteoporotic compression fractures $(n=5)$, myeloma $(n=2)$, and hemangioma $(n=1)$. The total number of vertebrae treated was $n=61$. On average, 3.05 vertebrae per patient were treated (1-6); almost $80 \%$ of the treated vertebrae were localized between TH8 and L2 (48 of 61). In all patients, only a single rotational acquisition was performed, which was focused on the region where implant distribution and leakage were of most interest to visualize. Thus, some treated vertebrae were not included. The total number of vertebrae controlled with $3 \mathrm{D}$ acquisitions and CT was 52 (cervical: 4; thoracic: 26; lumbar: 22). For PVP in the lumbar or thoracic spine, a transpedicular access was chosen; for treatment of cervical vertebrae, an anterolateral approach was used (for a detailed description of the PVP procedure, see Refs. 2-4 and 9).

\section{Image Acquisition, Postprocessing, and Analysis}

Three-dimensional rotational images were acquired immediately after the PVP procedure, with the patient remaining in the prone position (lumbar and thoracic spine) and supine position (cervical spine) on the table. Patients who were awake were asked to hold their breath during image acquisition; in patients who were treated under general anesthesia, a short ventilation stop was performed. Images were acquired on a biplane $\mathrm{C}$-arc angiographic unit (BV3000; Philips Medical Systems, The Netherlands) that allows creation of $3 \mathrm{D}$ anatomy, with the anatomy to be imaged positioned in the system isocenter. The rotational scan covers $180^{\circ}$ of circular trajectory and is accomplished in $8 \mathrm{sec}$ with a frame rate of 25 pictures/sec. The field of view could be chosen from 17 to 38 $\mathrm{cm}$ with a matrix of $1024 \times 1024$. To obtain images optimized for bone display, a dedicated acquisition system program was selected ("bone run"). Compared to the usual acquisition scheme for angiographic images, lowered kilovolt figures were used to improve visualization of bone with low calcium content as found in osteoporosis, and the number of images acquired during the rotational scan was doubled to 200 in order to achieve a higher contrast resolution. The images from rotational acquisition were transferred to the commercially available postprocessing station through a conventional network connection. For the transfer of 200 images, 5-6 sec were required.
The reconstructed dataset was visualized with different display techniques such as MultiPlanarReformat (MPR), Shaded Surface Display (SSD), Volume Rendering (VRT), and Maximum Intensity Projection (MIP). With these display techniques, it was possible either to display a 3D model of the entire volume or to analyze image slabs in three perpendicular views with the possibility to cross-reference the images.

Computed tomography scanning was performed on a helical MD-CT with a collimation of $1.5 \mathrm{~mm}$. Volume data was visualized on a workstation using 2D MPR reconstructions in three orthogonal planes.

The image analysis was done in conjunction by two experienced neuroradiologists. The analysis of the rotational acquisition images was performed prior to CT imaging. Images were evaluated directly at the postprocessing station with all image display modalities available. Evaluated parameters were, first, the presence, the degree (mild, severe diagnostic, severe nondiagnostic), and the assumed cause of any artifacts; second, the presence of cement leakage at different sites: posterior to the vertebra, paravertebral (anterior or lateral to the vertebral body), foraminal, and in the region of the vessels; third, it was noted which display technique was found most valuable to evaluate the individual vertebrae.

The postinterventional CT examination was the standard of reference for leakage. In a final site-by-site analysis, it was assessed whether CT images or rotational images were superior to assess implant distribution in relation to focal lesions (metastasis or hemangioma) of the vertebral bodies.

\section{Results}

The time from rotational image acquisition to visualization of 3D volume images was in all cases less than $10 \mathrm{~min}$. Artifacts occurred in three patients; in all cases, the artifacts were mild and caused by motion.

On CT images, leakage was found in 33 of 52 vertebrae, with most frequent localization being paravertebral $(50 \%)$. The findings on CT and rotational images agreed for the assessment in 30 vertebrae (Fig. 1). Five leakages detected on CT were missed (false negative) on images obtained by rotational acquisition and four were false positive detected with 3D rotational acquisition. Sensitivity and specificity for detecting the leakages with the 3D rotational image acquisition were 0.86 and 0.82 , respectively. In all cases, missed extravasations of cement were extremely small, and after analysis of the CT images, re-evaluation of the rotational images using the MIP display and thin slabs disclosed the leakage site (Fig. 2). Clinical symptons caused by the paravertebral leakage were recognized in only one patient with a transient radiculopathy. This leakage was readily detected (Fig. 1). False-positive results of cement leakage on rotational images were in all cases due to the fact that minimal amounts of cement were regarded not to be confined by bone, although on CT, cortical bone structures were detected (Fig. 3).

In all cases, the MIP slabs were regarded to be the best display technique to assess implant distribution. However, even with this image display technique, the assessment of 

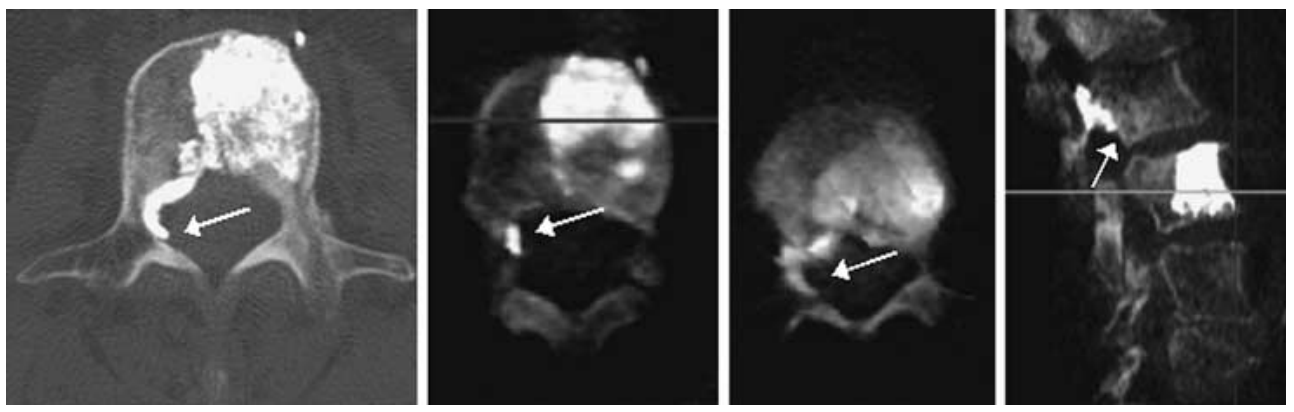

Fig. 1. A 65-year-old female with metastasis; treatment $\mathrm{L} 2$ and $L 3$; radiculopathy after PVP (transitory). CT left image. Leakage L2: foraminal, posterior, and paravertebral correctly recognized (arrow) on rotational acquisition (RA).
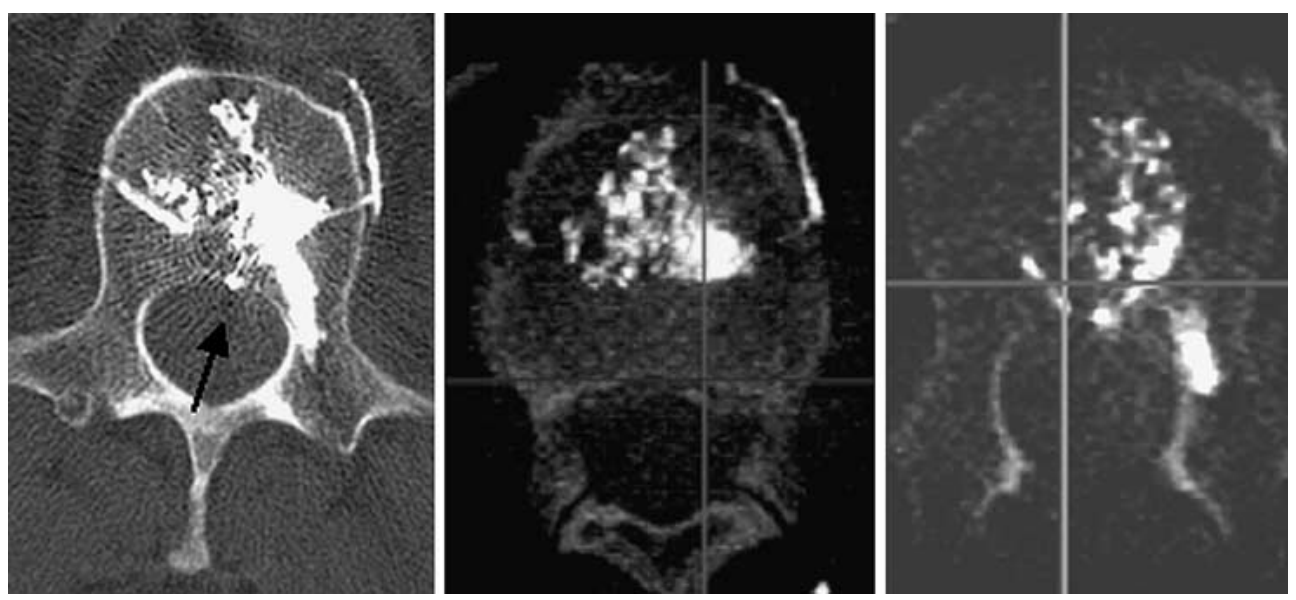

Fig. 2. A 71-year-old male with metastasis; treatment T11 to L1. CT left image. Leakage L1: paravertebral missed on RA initially; posterior leakage correctly recognized on RA (arrows).
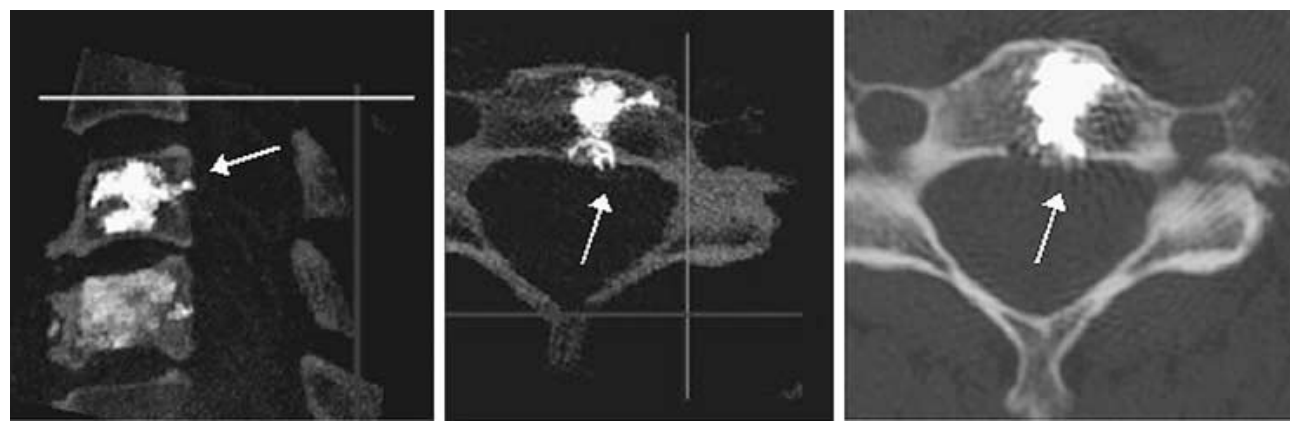

Fig. 3. A 82-year-old female with metastasis; treatment $\mathrm{C5}$ and C6. CT right image. posterior leakage of C5 false positive on RA.

implant distribution within focal lesions was found to be superior on CT images in all cases.

\section{Discussion}

Rotational image acquisition with a C-arc fluoroscopic unit is an established method in vascular $3 \mathrm{D}$ imaging but showed also to provide high-resolution multiplanar images and 3D volumes of cement distribution after vertebroplasty. In contrast to image acquisition techniques optimized for vascular imaging, a protocol that included large rotation angles with an increased number of projection radiographs per rotation ("bone run") was used.

With this approach, an assessment of implant distribution on volumetric images was possible in all 20 consecutive patients. Mild image artifacts (attributable to patient mo- tion) were present in only three patients. For image evaluation, MIP reconstructions displayed as slab images were found to be the preferred visualization modality in our setup.

What are the indications to perform a rotational image acquisition after PVP? It is well known that cement leakage is a common finding after PVP $[10,11]$. From our experience, projective fluoroscopy images are sufficient to control clinically significant cement leakages after the PVP procedure in the vast majority of patients. However, it can be difficult to exactly localize cement leakage on projective fluoroscopic images only. Especially in patients who are under general anesthesia and in whom cement leakage to critical anatomic structures (e.g., the neuroforamen) is suspected, it is of importance to have exact image information for the further workup of the patient $[6,11-13]$. The possibility of obtaining a 3D image dataset immediately after the 
cement injection (while the patient is still on the table) with the same X-ray equipment as used for the injection procedure is obviously of advantage to accelerate the therapeutic decision process and to initiate protective procedures after a complication. For example, as a therapeutic option in cases with cement leakage adjacent to nerve roots, Kelekis et al. described cooling of nerve roots through saline injection, which might help prevent heat irritation caused during the polymerization of the cement [14]. Three-dimensional acquisitions might here be of help to accurately determine the site of leakage, although in these cases an even faster acquisition procedure of the images would be of advantage.

The feasibility of obtaining volumetric images immediately after an intervention in the angio suite is clearly the key advantage of rotational acquisitions in comparison to CT imaging. On the contrary, the contrast resolution of the rotational images was clearly inferior compared to CT. Furthermore, the outlines of bones could be less distinctly detected with the rotational image acquisition system. The inferior depiction of the bone outlines on rotational images - as observed by other investigators [15, 16] — was the reason for false-positive findings of cement leakage in all of our cases. The use of improved reconstruction kernels, as are used with $\mathrm{CT}$, might improve the contrast resolution in rotational images, but this will have to be investigated in prospect studies.

Radiation exposure to the patient in rotational image acquisition compared to CT and fluoroscopy was not investigated in our study. Linsenmaier et al. described a considerable radiation dose reduction of approximately $50 \%$ in rotational image acquisition compared to standard CT protocols of the peripheral joints [15]. Nevertheless, comparative studies are needed to clarify the extent of radiation exposure to the patient involving the spinal column.

\section{Conclusion}

From our preliminary experiences, 3D rotational image acquisition can provide a straightforward method for postprocedural assessment of vertebroplasty procedures in patients with general anesthesia or complicated cement injection. Detection of cement leakage with a rotational image acquisition achieved an acceptable sensitivity compared to $\mathrm{CT}$ as the gold standard. Image reconstruction and image display are accurate. Additionally, rotational image acquisition could become of value in the pretherapeutic workup of the patients (e.g., if only cross-sectional magnetic resonance images are available and no CT scans for evaluation of the bone architecture).

\section{References}

1. Galibert P, Deramond H, Rosat P, et al. (1987) [Preliminary note on the treatment of vertebral angioma by percutaneous acrylic vertebroplasty]. Neurochirurgie 33(2):166-168

2. Martin JB, Jean B, Sugiu K, et al. (1999) Vertebroplasty: clinical experience and follow-up results. Bone 25(2 Suppl):11S-15S

3. Cotten A, Boutry N, Cortet B, et al. (1998) Percutaneous vertebroplasty: state of the art. Radiographics 18(2):311-320; discussion 320323

4. Kallmes DF, Jensen ME (2003) Percutaneous vertebroplasty. Radiology 229(1):27-36

5. McGraw JK, Cardella J, Barr JD, et al. (2003) Society of interventional radiology quality improvement guidelines for percutaneous vertebroplasty. J Vasc Interv Radiol 14(9 Pt 2):S311-S315

6. Gangi A, Kastler BA, Dietemann JL (1994) Percutaneous vertebroplasty guided by a combination of CT and fluoroscopy. Am J Neuroradiol 15(1):83-86

7. Hochmuth A, Spetzger U, Schumacher M (2002) Comparison of threedimensional rotational angiography with digital subtraction angiography in the assessment of ruptured cerebral aneurysms. Am J Neuroradiol 23(7):1199-1205

8. Gailloud P, Oishi S, Carpenter J, et al. (2004) Three-dimensional digital angiography: new tool for simultaneous three-dimensional rendering of vascular and osseous information during rotational angiography. Am J Neuroradiol 25(4):571-573

9. Martin JB, Gailloud P, Dietrich PY, et al. (2002) Direct transoral approach to $\mathrm{C} 2$ for percutaneous vertebroplasty. Cardiovasc Intervent Radiol 25(6):517-519

10. Garfin SR, Yuan HA, Reiley MA (2001) New technologies in spine: Kyphoplasty and vertebroplasty for the treatment of painful osteoporotic compression fractures. Spine 26(14):1511-1515

11. Cotten A, Dewatre F, Cortet B, et al. (1996) Percutaneous vertebroplasty for osteolytic metastases and myeloma: Effects of the percentage of lesion filling and the leakage of methyl methacrylate at clinical follow-up. Radiology 200(2):525-530

12. Mathis JM, Barr JD, Belkoff SM, et al. (2001) Percutaneous vertebroplasty: A developing standard of care for vertebral compression fractures. Am J Neuroradiol 22(2):373-381

13. Harrington KD (2001) Major neurological complications following percutaneous vertebroplasty with polymethylmethacrylate: A case report. J Bone Joint Surg Am 83-A(7):1070-1073

14. Kelekis AD, Martin JB, Somon T, et al. (2003) Radicular pain after vertebroplasty: compression or irritation of the nerve root? Initial experience with the "cooling system." Spine 28(14):E265-E269

15. Linsenmaier U, Rock C, Euler E, et al. (2002) Three-dimensional CT with a modified C-arm image intensifier: Feasibility. Radiology 224(1):286-292

16. El-Sheik M, Heverhagen JT, Alfke H, et al. (2001) Multiplanar reconstructions and three-dimensional imaging (computed rotational osteography) of complex fractures by using a C-arm system: initial results. Radiology 221(3):843-849 